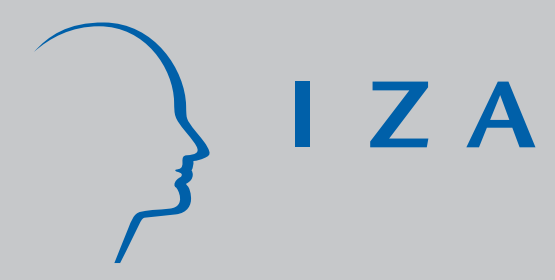

IZA DP No. 2256

Towards a Unifying Approach of the 'New Economic Geography'

Michael Pflüger

J ens Südekum

August 2006 


\title{
Towards a Unifying Approach of the 'New Economic Geography'
}

\author{
Michael Pflüger \\ University of Passau, \\ DIW Berlin and IZA Bonn
}

Jens Südekum

University of Konstanz

and IZA Bonn

\section{Discussion Paper No. 2256 \\ August 2006}

\author{
IZA \\ P.O. Box 7240 \\ 53072 Bonn \\ Germany \\ Phone: +49-228-3894-0 \\ Fax: +49-228-3894-180 \\ Email: iza@iza.org
}

\begin{abstract}
Any opinions expressed here are those of the author(s) and not those of the institute. Research disseminated by IZA may include views on policy, but the institute itself takes no institutional policy positions.

The Institute for the Study of Labor (IZA) in Bonn is a local and virtual international research center and a place of communication between science, politics and business. IZA is an independent nonprofit company supported by Deutsche Post World Net. The center is associated with the University of Bonn and offers a stimulating research environment through its research networks, research support, and visitors and doctoral programs. IZA engages in (i) original and internationally competitive research in all fields of labor economics, (ii) development of policy concepts, and (iii) dissemination of research results and concepts to the interested public.
\end{abstract}

IZA Discussion Papers often represent preliminary work and are circulated to encourage discussion. Citation of such a paper should account for its provisional character. A revised version may be available directly from the author. 


\section{ABSTRACT}

\section{Towards a Unifying Approach of the 'New Economic Geography'*}

Models of the new economic geography share a number of common conclusions, but also exhibit notable differences, in particular with respect to the shape of the location pattern and the efficiency of the market equilibrium. This reflects the fact that these models rely heavily on specific functional forms. In this paper we approach the properties of the 'footloose entrepreneur' class of new economic geography models with a unifying framework based on the indirect utility function of mobile agents. This approach has several payoffs. We are able to provide general, yet handy, formulae to determine the break point, the bifurcation pattern and the welfare properties of the market equilibrium. Moreover, an application of this framework allows us to show how specific results in the literature can be reconciled as special cases, thereby allowing us to highlight the origin of their differences.

JEL Classification: R12, R50, F12, F15, F22

Keywords: new economic geography, agglomeration, location pattern, regional policy

Corresponding author:

Jens Suedekum

University of Konstanz

Department of Economics

Fach D 132

78457 Konstanz

Germany

E-mail: jens.suedekum@uni-konstanz.de

\footnotetext{
* Special thanks to Jacques-Francois Thisse for extensive discussion with us on this subject matter. Moreover, we thank Kristian Behrens, Johannes Bröcker, Georg Hirte, Pierre Picard, Michael Rauscher, Klaus Schöler, Marcel Thum, Klaus Wälde and participants of seminars at CORE Louvain, Dresden, Frankfurt, Göttingen and Regensburg for helpful comments and suggestions. The usual disclaimer applies.
} 


\section{Introduction}

Economists have rediscovered their interest for spatial issues in recent years. This revival owes much to the emergence of the 'new economic geography'. The development of the coreperiphery model by Krugman (1991) was followed up by numerous analyses which extended his basic framework. More recently, building on these models there was an explosion of work which addresses policy issues in the fields of trade, taxation and regional economics and which has started to put the welfare properties of the market equilibrium under scrutiny. ${ }^{1} \mathrm{New}$ economic geography models share a number of properties. One crucial property is the prediction that, due to market size effects ('linkages', 'pecuniary externalities'), an asymmetric spatial structure may emerge endogenously. Another one is the notion that, because of these pecuniary externalities, the spatial allocation of firms resulting in the market equilibrium is not efficient in general. However, a more detailed inspection reveals that new economic geography models exhibit notable differences, too. In this paper we address two key differences of these models, their predictions concerning the shape of the location pattern (i.e. the 'bifurcation pattern') and the efficiency of the market equilibrium.

The aim of this paper is to provide an integrative treatment of the determination of the location pattern and of the efficiency issue. A fully general analysis is beyond the scope of this paper (and is presumably out of reach), the reason being that the new economic geography builds on the assumption that firms produce under increasing returns and do have market power. The quest for a general model of imperfect competition has (so far) been elusive, however. Therefore, we confine our attention to a subclass of new economic geography models, the so-called 'footloose entrepreneur-models', in which agglomeration is the result of mobile consumers which render the local market size endogenous. ${ }^{2}$ However, this limitation is less restrictive than it might appear at a first glance, since footloose entrepreneur models are tied up one to one with the standard core-periphery framework of Krugman (1991) through the analysis provided by Forslid and Ottaviano (2003). Moreover, the properties of Krugman's model are replicated by an alternative class of new economic geography models which focus on vertical linkages between firms (Ottaviano and Robert-Nicoud 2006).

\footnotetext{
${ }^{1}$ The most comprehensive treatments to date can be found in the monographs of Fujita et al. (1999), Fujita and Thisse (2002) and Baldwin et al. (2003) and in the survey by Ottaviano and Thisse (2004).

${ }^{2}$ The term 'footloose entrepreneur models' was coined by Baldwin et al. (2003) and comprehends analytically tractable models of the 'labour mobility class' of new economic geography models which originated in Krugman's core-periphery model (see e.g. Ottaviano and Thisse 2004). The footloose entrepreneur approach is due to independent research by Forslid and Ottaviano, later published as Forslid and Ottaviano (2003). Venables (1996) and Krugman and Venables (1995) have developed an alternative 'vertical linkages framework' in which agglomeration is the result of input-output linkages among firms (Ottaviano and Thisse 2004, Ottaviano and Robert-Nicoud 2006).
} 
Our approach differs from the usual one: rather than starting with specific functional forms for individual preferences, we present a generalised framework based on the indirect utility function of mobile agents. This approach has several payoffs. We are able to provide general, yet handy, formulae to determine the break point, the bifurcation pattern and the welfare properties of the market equilibrium. An application of this framework allows us to show how specific results in the literature can be reconciled as special cases, thereby allowing us to highlight the origin of their differences. With this unifying approach we hope to shed some light on the robustness of results that have been provided by the new economic geography and to strengthen their scope and applicability for policy purposes.

In the rest of this section, we review the literature and point out the key differences that obtain across models. In section 2 we introduce our unifying framework. Sections 3 and 4 apply this framework to the class of footloose-entrepreneur models and highlight the origin of their differences with respect to their location pattern and welfare properties. The concluding section provides a brief summary and outlook.

The new economic geography has relied heavily on specific functional forms. A key difference concerns the specification of individual preferences. The core-periphery model by Krugman (1991) assumes an upper-tier uility function of the Cobb-Douglas-type and CESsubpreferences over manufacturing varieties. The same preference structure is assumed in the footloose entrepreneur model by Forslid and Ottaviano (2003), an analytically tractable version of the former which mimicks all of its essential features (Robert-Nicoud 2005). The bifurcation pattern implied in a trade integration process in this CP-model is such that symmetry is the only stable location equilibrium for high levels of trade costs. Once trade costs have fallen to a critical level, the 'break point', all mobile economic activity concentrates in a single region. This model exhibits hysteresis. A reversal of trade costs need not restore symmetry, because the core-periphery pattern is stable up to a 'sustain point' which obtains at a strictly higher level of trade costs than the break point. In contrast, in the footloose entrepreneur model by Ottaviano, Tabuchi and Thisse (2002), where the upper-tier utility is a quadratic quasi-linear function, the break point and the sustain point coincide. Hence, this model, which we term QLQuad-model, predicts catastrophic aggloeration without hysteresis. Yet other papers provide results that break even more drastically with the idea of catastrophic agglomeration. The footloose entrepreneur model by Pflüger (2004) which assumes a logarithmic quasi-linear upper-tier utility, predicts a smooth transition from symmetry to agglomeration in the course of trade integration. Hence, in this QLLog-model, there are stable 
interior equilibria with some, but not all mobile workers concentrated in one region. Figure 1 summarises the location pattern of these three models.

[Figure 1 about here]

With regard to the efficiency question, a general finding is that the equilibrium pattern need not coincide with the socially optimal spatial structure. This is due to the fact that the models of the new economic geography are replete with pecuniary externalities, which matter for aggregate welfare under imperfect competition. However, beyond this general insight, the model predictions differ. Under the commonly used normative criterion of a utilitarian social welfare function that the planner is assumed to maximise subject to market prices (secondbest optimum), partly contradictory normative conclusions obtain. Ottaviano, Tabuchi and Thisse (2002) and Pflüger and Südekum (2006) find that the market outcome always implies over-agglomeration around the break point. Baldwin et al. (2003: ch.11), in contrast, show that, depending on the relative factor endowment of immobile households, there may be overor under-agglomeration at the break point in the CP-model by Forslid and Ottaviano (2003).

\section{$2 \quad$ A unifying framework}

\subsection{Basic assumptions}

This section presents our framework based on a general indirect utility function. We look at an economy composed of two regions (labelled 'home' and 'foreign') with identical tastes and technologies. There are two types of households with perfectly inelastic factor supply. The first type, call it 'skilled labour' or 'entrepreneurs' $(K)$ is mobile across regions. The second type $(L)$ is immobile and equally distributed across the two locations. Both household types derive utility from the consumption of two types of goods. There is an agricultural good $(A)$, which is homogeneous, traded without cost and produced competitively with a unit input requirement of $L$. This good serves as the numéraire and is assumed to be produced in both regions throughout the analysis. It follows from these assumptions that the wage (income) of the unskilled is fixed at unity in both regions. The second good is a manufacturing aggregate $X$ that consists of a large variety of differentiated products. Individual utility functions exhibit a 'love of variety effect', i.e. for a given income level, consumers achieve a higher

utility level the higher is the number of varieties available. Each manufacturing good is produced by a single firm under internal economies of scale with strictly positive fixed costs and positive marginal costs. The fixed cost component is due to the compensation of skilled 
labour $K$ of which one unit is needed to produce at all. Immobile unskilled labour $L$ is the only variable input in this industry. Each firm has a negligible impact on the aggregate market outcome and there is no strategic interaction between firms. Due to entry and exit, firms make zero profits. Hence, the operating profits are the rents (wage incomes) that accrue to skilled labour. Trade of manufacturing varieties across regions is inhibited by trade costs which are measured by some parameter $\tau$ or by some measure of trade freeness $\phi$.

\subsection{Indirect utility function and the equation of motion}

The long-run spatial equilibrium is determined by the migration decision of mobile agents. They respond to differentials in (indirect) utility levels across locations. In contrast to previous analyses we do not specify individual preferences. We simply assume the existence of a utility function with standard continuity and non-satiation properties which gives rise to an indirect utility function of the form (Mas-Colell et al. 1995: 56f.):

$$
V=V\left(Y(\lambda, \tau, \cdot), P(\lambda, \tau, \cdot), p_{A}, \cdot\right)
$$

The utility of mobile workers living in the domestic region depends on attainable income $Y$ and on consumer prices $P$ and $p_{A}=1$ in that location. The price of the numéraire good is equal to one and is henceforth suppressed. With some loss of generality we assume that $P$ is a scalar which captures consumer prices in a single number. ${ }^{3}$ By analogy, indirect utility in the foreign region (distinguished by an asterisk $*$ ) is given by $V^{*}=V^{*}\left(Y^{*}, P^{*}\right){ }^{4}$ We make the following assumptions. Income and prices can be described by functions $Y(\cdot)$ and $P(\cdot)$ that depend on the endogenous share $0<\lambda<1$ of mobile workers located in the domestic region, on the exogenous level of transport costs $\tau$, and on the other primitives of the model. Both functions $Y(\cdot)$ and $P(\cdot)$ are differentiable with respect to $\lambda$. Furthermore, $V(\cdot)$ is assumed to be continuous and differentiable with respect to both arguments. Letting subscripts denote partial derivatives, and suppressing the arguments of $V(\cdot)$, we have $V_{Y}>0$, $V_{P}<0, V_{Y^{*}}^{*}>0, V_{P^{*}}^{*}<0$ (Mas-Colell 1995). ${ }^{5}$

\footnotetext{
${ }^{3}$ In general, $P$ is a vector of consumer prices. Under standard separability assumptions on the utility function, this vector can be aggregated into a scalar (e.g. Varian 1992: 147ff.). These assumptions are fulfilled in the preexisting models of the new economic geography, notably when sub-preferences are of the CES-type. An example where $P$ is not a scalar is the more recent CARA-model of Behrens and Murata (2006).

${ }^{4}$ Since the agricultural good is assumed to be produced in both regions and can be traded without costs, the law of one price implies that its price is unity in the foreign region as well.

${ }^{5}$ We assume a strictly negative response of the indirect utility with respect to the manufacturing price scalar.
} 
Using the common assumption of myopic adjustment (see Baldwin 2001, or Ottaviano and Thisse 2004 for a critical assessment), the equation of motion for mobile workers is given by

$$
\frac{d \lambda}{d t} \equiv \dot{\lambda}=\left(V(\cdot)-V^{*}(\cdot)\right) \cdot \lambda \cdot(1-\lambda)
$$

There are two types of equilibria in this model, interior ones with $V(\cdot)=V^{*}(\cdot)$ where either symmetry $\left(\lambda=\frac{1}{2}\right)$ or stable partial agglomeration $\left(0<\lambda<1, \lambda \neq \frac{1}{2}\right)$ obtains, or core-periphery equilibria with $V(\cdot) \geq V^{*}(\cdot)$ and $\lambda=1$, or $V(\cdot) \leq V^{*}(\cdot)$ and $\lambda=0$.

\subsection{Determination of the bifurcation point}

Due to the symmetry of the two regions, an equal division of mobile workers across locations $\left(\lambda=\frac{1}{2}\right.$ ) is always an equilibrium which is assumed to prevail at initially high trade costs. This equilibrium is not necessarily stable, however, because there is an interaction of destabilising agglomeration forces and of stabilising dispersion forces. The stability of the symmetric equilibrium depends on the sign of $\partial\left(V(\cdot)-V^{*}(\cdot)\right) / \partial \lambda$ evaluated at $\lambda=\frac{1}{2}$. Of course, agglomeration forces may be so weak that symmetry never becomes unstable, or they may be so strong that symmetry becomes unstable even at infinitely high trade costs. Both cases are usually excluded by appropriate restrictions on the model primitives (e.g. the 'no black hole'condition). Assuming existence (i.e. ruling out $\partial\left(V(\cdot)-V^{*}(\cdot)\right) / \partial \lambda>0$ or $\partial\left(V(\cdot)-V^{*}(\cdot)\right) / \partial \lambda<0 \forall \tau, \phi$ at $\left.\lambda=\frac{1}{2}\right)$, there is a critical level of trade costs, the (market) 'break point' $\tau_{b}$, at which the symmetric equilibrium becomes unstable. This bifurcation point is formally implied by the condition

$$
\left.\left.\frac{\partial\left(V(\cdot)-V^{*}(\cdot)\right)}{\partial \lambda}\right|_{\lambda=1 / 2}=0 \Leftrightarrow \tau_{b} \quad \text { (equivalently, } \phi_{b}\right)
$$

There need not be a unique solution for $\tau$ in the relevant range. The lowest level of $\tau$ that satisfies eq. (3) is called the 'break point'. In the models that are summarised in figure 1, the bifurcation point is unique, i.e. there is only one value of $\tau$ such that (3) is satisfied. ${ }^{6}$

\footnotetext{
${ }^{6}$ One possibility to obtain more than one solution is to introduce a congestion force such as housing into the model (as in Tabuchi 1998, Ottaviano et al. 2002 and Pflüger and Südekum 2006). The consequence is that below some critical level of trade costs, symmetry reemerges as the unique stable equilibrium, i.e. eq. (3) is also satisfied at this 're-dispersion point'. We abstract from such congestion forces in the present analysis.
} 
Using eq. (1), the first derivative of the indirect utility differential $V(\cdot)-V^{*}(\cdot)$ is given by

$$
\frac{\partial\left(V(\cdot)-V^{*}(\cdot)\right)}{\partial \lambda}=V_{P} P_{\lambda}-V_{P^{*}}^{*} P_{\lambda}^{*}+V_{Y} Y_{\lambda}-V_{Y^{*}}^{*} Y_{\lambda}^{*},
$$

where all terms on the right hand side depend on $\lambda$ and $\tau$. Since regions have identical preferences, $V_{P^{*}}^{*}=V_{P}$ and $V_{Y^{*}}^{*}=V_{Y}$. Moreover, around $\lambda=\frac{1}{2}$ we have $P_{\lambda}^{*}=-P_{\lambda}$ and $Y_{\lambda}^{*}=-Y_{\lambda}$. Applying (3) at $\lambda=\frac{1}{2}$ we obtain:

$$
\left.\frac{\partial\left(V(\cdot)-V^{*}(\cdot)\right)}{\partial \lambda}\right|_{\lambda=1 / 2}=2\left(V_{P} P_{\lambda}+V_{Y} Y_{\lambda}\right)=0 \Leftrightarrow-\frac{V_{P}}{V_{Y}}=\frac{Y_{\lambda}}{P_{\lambda}}
$$

This condition captures the central forces of the new economic geography, a supply linkage as an agglomeration force which works through the effects of the number of firms on the price level, and hence on utility $\left(V_{P} P_{\lambda}\right)$, and a demand linkage and a competition effect which work through the influence of the number of firms on the wage that firms can just afford to pay to break even, and hence on utility $\left(V_{Y} Y_{\lambda}\right)$. The demand linkage is an agglomeration force and the competition effect is a dispersion force. Applying Roy's identity, condition (5) can also be written as $Y_{\lambda} / P_{\lambda}=C^{X} \geq 0$, where $C^{X}$ denotes demand for the aggregate of manufactures. This formulation shows that $P_{\lambda}$ and $Y_{\lambda}$ must have the same sign at the break point. In standard models, $P_{\lambda}$ is negative due to the supply linkage, as the price index declines when more firms locate in the domestic region. Hence, at $\tau_{b}$ income must also be decreasing in $\lambda$, i.e. $Y_{\lambda}<0$.

\section{$2.4 \quad$ The location pattern}

The bifurcation pattern that emerges at the break point can be determined by higher order derivatives of the indirect utility differential evaluated at $\lambda=\frac{1}{2}$ and the critical level of trade costs $\tau_{b}$ (e.g. Fujita et al. 1999; Grandmont 1988; Guckenheimer and Holmes 1983). A necessary condition for a bifurcation to occur is $\partial^{2}\left(V(\cdot)-V^{*}(\cdot)\right) /\left.\partial \lambda^{2}\right|_{\lambda=1 / 2, \tau=\tau_{b}}=0$, i.e. the second order derivative must be zero. Whether the bifurcation is a sub-critical pitchfork ('tomahawk') as in fig. 1a, a super-critical pitchfork ('pitchfork') as in fig. 1b, or the borderline case depicted in fig. 1c, is determined by the sign of the third order derivative, $\partial^{3}\left(V(\cdot)-V^{*}(\cdot)\right) / \partial \lambda^{3}$, which evaluated at $\lambda=\frac{1}{2}$ and $\tau=\tau_{b}$ depends only on exogenous model 
primitives. If this term is greater than (smaller than) zero, a tomahawk (pitchfork) bifurcation is implied, and the borderline case follows if the term is equal to zero. Differentiating (4) with respect to $\lambda$ yields

$$
\begin{aligned}
\frac{\partial^{2}\left(V(\cdot)-V^{*}(\cdot)\right)}{\partial \lambda^{2}}=\left(V_{P P} P_{\lambda}\right. & \left.+V_{P Y} Y_{\lambda}\right) P_{\lambda}+V_{P} P_{\lambda \lambda}-\left(V_{P^{*} P^{*}}^{*} P_{\lambda}^{*}+V_{P^{*} Y^{*}}^{*} Y_{\lambda}^{*}\right) P_{\lambda}^{*}-V_{P^{*}}^{*} P_{\lambda \lambda}^{*} \\
& +\left(V_{Y P} P_{\lambda}+V_{Y Y} Y_{\lambda}\right) Y_{\lambda}+V_{Y} Y_{\lambda \lambda}-\left(V_{Y^{*} P^{*}}^{*} P_{\lambda}^{*}+V_{Y^{*} Y^{*}}^{*} Y_{\lambda}^{*}\right) Y_{\lambda}^{*}-V_{Y^{*}}^{*} Y_{\lambda \lambda}^{*}
\end{aligned}
$$

Using the fact that preferences are identical across regions (i.e. $V_{P^{*}}^{*}=V_{P}, V_{Y^{*}}^{*}=V_{Y}$ and so forth) and taking into account that at $\lambda=\frac{1}{2}$ we have $P_{\lambda}^{*}=-P_{\lambda}, Y_{\lambda}^{*}=-Y_{\lambda}, P_{\lambda \lambda}^{*}=P_{\lambda \lambda}$ and $Y_{\lambda \lambda}^{*}=Y_{\lambda \lambda}$ due to symmetry, one immediately obtains the result that eq. (6) is equal to zero at $\lambda=\frac{1}{2}$, in general. Hence the second condition for a bifurcation is generally fulfilled.

Moving to the third order derivative, using $\lambda=\frac{1}{2}$, homogeneous preferences, and the fact that the cross derivatives of the utility function are identical (e.g. $V_{P Y}=V_{Y P}, V_{P Y Y}=V_{Y P P}$ and so forth), we obtain after straightforward if somewhat tedious manipulations

$$
\left.\Delta(\cdot) \equiv \frac{\partial^{3}\left(V(\cdot)-V^{*}(\cdot)\right)}{\partial \lambda^{3}}\right|_{\lambda=1 / 2, \tau=\tau_{b}}=\left.2 \cdot \frac{\partial^{3} V(\cdot)}{\partial \lambda^{3}}\right|_{\lambda=1 / 2, \tau=\tau_{b}}=2\left[\Lambda_{P}(\cdot)+\Lambda_{Y}(\cdot)+\Lambda_{Y P}(\cdot)\right]
$$

where

$$
\begin{aligned}
& \Lambda_{P}(\cdot)=\left[V_{P} P_{\lambda \lambda \lambda}+3 V_{P P} P_{\lambda} P_{\lambda \lambda}+V_{P P P}\left(P_{\lambda}\right)^{3}\right] \\
& \Lambda_{Y}(\cdot)=\left[V_{Y} Y_{\lambda \lambda \lambda}+3 V_{Y Y} Y_{\lambda} Y_{\lambda \lambda}+V_{Y Y Y}\left(Y_{\lambda}\right)^{3}\right] \\
& \Lambda_{Y P}(\cdot)=3\left[V_{Y P}\left(P_{\lambda \lambda} Y_{\lambda}+Y_{\lambda \lambda} P_{\lambda}\right)+V_{Y P P} Y_{\lambda}\left(P_{\lambda}\right)^{2}+V_{Y Y P} P_{\lambda}\left(Y_{\lambda}\right)^{2}\right]
\end{aligned}
$$

The shape of the location pattern depends on the sign of $\Delta(\cdot)$ which can be decomposed into three parts. The terms $\Lambda_{Y}$ and $\Lambda_{P}$ collect direct derivatives of $V(\cdot)$ with respect to the income and the price aggregator and derivates of $Y(\cdot)$ and $P(\cdot)$ with respect to $\lambda$. The term $\Lambda_{Y P}$ captures all cross-derivatives of $V(\cdot)$. This decomposition is useful in the comparison of models which we undertake below. 


\section{$2.5 \quad$ Welfare analysis}

The equilibrium allocation that results from the private location decision of mobile workers may not be optimal from a social point of view. This is so, because new economic geography models feature several distortions in parallel. First, firms do not price at marginal costs. Second, mobile entrepreneurs neglect the impact of their location decision on other agents. These effects are transmitted through market prices and therefore represent pecuniary externalities that have significance for total welfare under imperfect competition.

The normative criterion we consider is a simple utilitarian social welfare function. The planner maximises the un-weighted sum of utilities of all agents in the economy. Although this choice is not undisputed, in particular when the marginal utility of income is not constant and equal across agents, it is commonly used in the literature that addresses efficiency issue in new economic geography models. ${ }^{7}$ This justifies its use in our model comparison. We consider the second-best optimum where the planner is not able to impose marginal cost pricing, but complies to market prices when maximizing the social welfare function $\Omega(\lambda){ }^{8}$ This function is given by

$$
\Omega(\lambda)=K\left(\lambda V(\cdot)+(1-\lambda) V^{*}(\cdot)\right)+(L / 2)\left(v(\cdot)+v^{*}(\cdot)\right)
$$

where $K$ and $L$ are the total stocks of mobile and immobile households, respectively, and $v(\lambda, \tau), v^{*}(\lambda, \tau)$ is indirect utility of an immobile worker in the domestic (foreign) region. Differentiating (8) yields:

$$
\frac{\partial \Omega(\cdot)}{\partial \lambda}=K\left(\lambda \cdot \frac{\partial V(\cdot)}{\partial \lambda}+(1-\lambda) \frac{\partial V^{*}(\cdot)}{\partial \lambda}\right)+K\left(V(\cdot)-V^{*}(\cdot)\right)+\frac{L}{2} \cdot\left(\frac{\partial v(\cdot)}{\partial \lambda}+\frac{\partial v^{*}(\cdot)}{\partial \lambda}\right)
$$

It is straightforward to show that (9) is always equal to zero at $\lambda=\frac{1}{2}$. Hence, the social welfare function $\Omega(\cdot)$ always has an extremum at a symmetric allocation of firms. However, this may be a welfare maximum or a welfare minimum. In order to proceed with a local marginal analysis around the symmetric configuration we have to impose further restrictions on preferences. In particular we assume that preferences are such that (i) the symmetric configuration $\lambda=\frac{1}{2}$ is a unique global welfare maximum of the social welfare function for high trade costs only, (ii) that over the whole range of $\tau$ the symmetric configuration turns

\footnotetext{
${ }^{7}$ An analysis of other welfare criteria in relation to the core-periphery model is provided in Charlot et al. (2005).

${ }^{8}$ It is straightforward to characterise the first-best solution in our framework. Moreover, a condition can be derived under which the first-best and the second-best optimum coincide. We supply the analysis on request.
} 
from being a welfare maximum at high trade costs to being a welfare minimum at very low trade costs only once and remains a welfare minimum as $\tau \rightarrow 1$, and (iii) that the function $\Omega(\lambda, \cdot)$ has at most three internal extrema over the range $0<\lambda<1$. These assumptions limit the generality of our analysis. However, these properties are satisfied for the specific models that we consider below (see figure 2 below). Hence, they serve to achieve our aim which is to integrate existing and conflicting results which have been derived in the literature.

Under these additional assumptions we can use the second derivative of the social welfare function to determine whether the equilibrium allocation emerging at the 'break point' $\tau_{b}$ is socially optimal in the sense that symmetry turns from being a social welfare maximum to being a minimum at exactly this trade cost level. If the second derivative of the social welfare function is positive (negative) at $\lambda=1 / 2$ and $\tau=\tau_{b}$, symmetry is a local welfare minimum (maximum) at the market break point. The condition $\partial^{2} \Omega / \partial \lambda^{2}=0$ defines the 'social break point' $\tau_{p}$. This second derivative is given by:

$$
\frac{\partial^{2} \Omega(\cdot)}{\partial \lambda^{2}}=\lambda K\left(\frac{\partial^{2}\left(V(\cdot)-V^{*}(\cdot)\right)}{\partial \lambda^{2}}\right)+2 K\left(\frac{\partial\left(V(\cdot)-V^{*}(\cdot)\right)}{\partial \lambda}\right)+K\left(\frac{\partial^{2} V^{*}(\cdot)}{\partial \lambda^{2}}\right)+\frac{L}{2} \cdot\left(\frac{\partial^{2} v(\cdot)}{\partial \lambda^{2}}+\frac{\partial^{2} v^{*}(\cdot)}{\partial \lambda^{2}}\right)
$$

From (5) and (6) we know $\partial\left(V(\cdot)-V^{*}(\cdot)\right) / \partial \lambda=2\left[V_{Y} Y_{\lambda}+V_{P} P_{\lambda}\right]$ and $\partial^{2}\left(V(\cdot)-V^{*}(\cdot)\right) / \partial \lambda^{2}=0$ with $\lambda=\frac{1}{2}$. We can also derive:

$$
\begin{gathered}
\frac{\partial^{2} V(\cdot)}{\partial \lambda^{2}}=\frac{\partial^{2} V^{*}(\cdot)}{\partial \lambda^{2}}=\left[V_{P} P_{\lambda \lambda}+V_{P P} \cdot\left(P_{\lambda}\right)^{2}\right]+\left[V_{Y} Y_{\lambda \lambda}+V_{Y Y} \cdot\left(Y_{\lambda}\right)^{2}\right]+2 V_{P Y} P_{\lambda} Y_{\lambda} \\
\frac{\partial^{2} v(\cdot)}{\partial \lambda^{2}}+\frac{\partial^{2} v^{*}(\cdot)}{\partial \lambda^{2}}=2\left[V_{P} P_{\lambda \lambda}+2 V_{P P} \cdot\left(P_{\lambda}\right)^{2}\right]
\end{gathered}
$$

where we have made use of the fact $Y_{\lambda}=Y_{\lambda \lambda}=0$ for immobile workers because their income (wage) is fixed at a level of unity (cf. section 2.1), and that $v_{P}=v_{P}^{*}=V_{P}, v_{P P}=V_{P P}$ due to homogeneous preferences. Substituting (11) and (12) into (10), and using $\rho \equiv L / K$ we obtain

$$
\left.\frac{\partial^{2} \Omega(\cdot)}{\partial \lambda^{2}}\right|_{\lambda=1 / 2}=4 K\left[V_{Y} Y_{\lambda}+V_{P} P_{\lambda}\right]+\Psi(\cdot)
$$

where $\quad \Psi(\cdot)=K\left[(1+\rho)\left(V_{P} P_{\lambda \lambda}+V_{P P} \cdot\left(P_{\lambda}\right)^{2}\right)+\left(V_{Y} Y_{\lambda \lambda}+V_{Y Y} \cdot\left(Y_{\lambda}\right)^{2}\right)+\left(2 V_{Y P} Y_{\lambda} P_{\lambda}\right)\right]$ 
The market allocation is governed solely by the first term in (13), which contains the familiar break point condition (5). As we have already shown, this term is equal to zero at $\lambda=\frac{1}{2}$ and $\tau=\tau_{b}$. However, this 'market break point' coincides with the 'social break point' $\tau_{p}$ where $\partial^{2} \Omega / \partial \lambda^{2}=0$ if and only if $\Psi(\cdot)=0$. The term $\Psi($.$) can be understood as the 'net pecuniary$ externality' which captures the (quantitative) difference between the market's tendency to agglomerate and the social desirability of agglomeration. This function can be partitioned into a part that depends on the cross-derivative of $V(\cdot)$, i.e. the term $2 V_{Y P} Y_{\lambda} P_{\lambda}$, and two parts that depend on the direct derivatives only. If $\Psi(\cdot)$ is positive at $\lambda=\frac{1}{2}$ and $\tau=\tau_{b}$ the market delivers under-agglomeration at the 'market break point', because $\partial^{2} \Omega /\left.\partial \lambda^{2}\right|_{\lambda=1 / 2}>0$ in this case, which implies that the social planner would already have chosen (partial or full) agglomeration rather than a symmetric configuration. If the term $\Psi(\cdot)$ is negative at $\lambda=\frac{1}{2}$ and $\tau=\tau_{b}$ there is instead market over-agglomeration.

Figure 2 illustrates our welfare analysis by depicting the shapes the welfare function $\Omega(\cdot)$ can take over the full range of $\lambda$ for different values of trade costs $\tau$ and for the three specific models that we study in our application section 3 below. In graphical terms our analysis amounts to studying the behaviour of $\Omega(\cdot)$ at $\lambda=\frac{1}{2}$.

\section{[Figure 2 about here]}

Figure 2 points at one caveat which applies to our welfare analysis. In the CP-model by Forslid and Ottaviano (2003), the social welfare function can exhibit an 'W-shape' as depicted in panel a3. Symmetry is a local but not a global welfare maximum in this case. Hence, the condition $\Psi()<$.0 does not suffice to conclude that there is market over-agglomeration. Rather, in addition one has to check whether $\Omega\left(\lambda=\frac{1}{2}\right)>\Omega(\lambda=1)=\Omega(\lambda=0)$ at $\tau=\tau_{b}$ to conclude that the market actually over-agglomerates. In other words, the term $\Psi($.$) is a local$ measure which may wrongly signal over-agglomeration when agglomeration is in fact socially desirable. However, under the additional assumptions (i) - (iii) which we have imposed, the direction of this signal is unambiguous. In particular, the inverse case is never possible. When applying our methodology to specific cases, we have to check this additional condition. Before turning to applications, we briefly summarise the results from this section. 


\section{Summary of results}

In the unifying footloose entrepreneur model where the location choice of mobile households is governed by the indirect utility differential $V(P(\cdot), Y(\cdot))-V^{*}\left(P^{*}(\cdot), Y^{*}(\cdot)\right)$ the following results hold:

(1) Provided a break point exists, it is determined by the condition $-V_{P} / V_{Y}=Y_{\lambda} / P_{\lambda}$.

(2) Around the break point, the wage of the skilled falls when more firms move into a region as long as $P_{\lambda}<0$ (the demand linkage is dominated by the competition effect).

(3) The bifurcation pattern that unfolds at the break point is determined by the term $\Delta\left(\lambda=\frac{1}{2}, \tau=\tau_{b}\right)$, given by eq. (7). If this term is positive (negative), the model exhibits a tomahawk (pitchfork) bifurcation. For $\Delta(\cdot)=0$, the borderline case is implied.

(4) Under the assumption that the allocation of mobile agents $\lambda$ is chosen so as to maximise a utilitarian social welfare function subject to market prices, the term $\Psi\left(\lambda=\frac{1}{2}, \tau=\tau_{b}\right)$ given by eq. (14) indicates a net pecuniary externalitiy. If it is negative (positive) the market delivers over- (under-) agglomeration at the break point. The 'market break point' coincides with the 'social break point' if $\Psi\left(\lambda=\frac{1}{2}, \tau=\tau_{b}\right)=0$. When the net pecuniary externality is negative and the social welfare function exhibits an 'W-shape' it has to be checked in addition whether $\Omega\left(\lambda=\frac{1}{2}\right)>\Omega(\lambda=1)=\Omega(\lambda=0)$ at $\tau=\tau_{b}$ in order to verify that there is market over-agglomeration.

\section{Applications}

In this section we apply our framework to the three footloose entrepreneur models which we have introduced in the review of the literature: the core-periphery (CP) model, the quasi-linear logarithmic (QLLog) model, and the quasi-linear quadratic (QLQuad) model. Analytical details for these three models are reported in appendices A1, A2 and A3, respectively.

\subsection{The CP-model}

The footloose entrepreneur model by Forslid and Ottaviano (2003) is the analytically tractable version of the core-periphery (CP) model by Krugman (1991). This model features a CobbDouglas upper-tier utility function $U(X, A)=X^{\mu} A^{1-\mu}, 0<\mu<1$, with CES preferences for the single manufacturing varieties. Consumer prices of the symmetrical varieties differ across regions by a multiplicative constant due to the assumption of 'iceberg' trade costs. Hence, the price aggregator $P(\cdot)$ is a scalar which is given by the CES price index and which takes account of trade costs through a standard measure of trade freeness $0<\phi \equiv \tau^{1-\sigma}<1$ where $\sigma>1$ denotes the constant elasticity of substitution between any two manufacturing varieties. The Cobb-Douglas preference structure implies an indirect utility function of the form 
$V(\cdot)=Y(\lambda, \phi) \cdot(P(\lambda, \phi))^{-\mu}$. Hence, $V_{Y Y}=V_{Y Y Y}=V_{Y Y P}=0$. Using the expressions for $Y(\lambda, \phi)$ and $P(\lambda, \phi)$ from Forslid and Ottaviano (2003), taking derivatives $Y_{\lambda}$ and $P_{\lambda}$, using $\lambda=\frac{1}{2}$ and applying (5) yields the break point $\phi_{b, C P}$ as reported by Forslid and Ottaviano.

Turning to the shape of the location pattern and the welfare properties around the break point, we insert the functional forms of this model in eqs. (7) and (14), respectively. We then apply $\lambda=\frac{1}{2}$ and $\phi=\phi_{b, C P}$, and rearrange terms to obtain expressions for $\Delta_{C P}\left(\frac{1}{2}, \phi_{b, C P}\right)$ and $\Psi_{C P}\left(\frac{1}{2}, \phi_{b, C P}\right)$. The term $\Delta_{C P}\left(\frac{1}{2}, \phi_{b, C P}\right)$ is unambiguously positive. This verifies that the CP model exhibits a tomahawk bifurcation as depicted in fig. 1a. The sign of $\Psi_{C P}\left(\frac{1}{2}, \phi_{b, C P}\right)$ is ambiguous and depends on the relative endowment of the immobile factor, $\rho$. If $\rho$ is large the term is negative and the market exhibits over-agglomeration at the break point. Conversely, if $\rho$ is small the market delivers too little agglomeration. This ambiguity of the welfare properties corresponds one to one with the conclusion of Baldwin et al. (2003: sect.11.3.) who have performed a second-best global welfare analysis for the CP-model by Forslid and Ottaviano (2003). Hence, we can conclude that the net pecuniary externality term $\Psi($.$) provides the qualitatively correct signal and can therefore be utilised for the model$ comparison that we undertake in section 4.

\subsection{The QLLog-model}

The QLLog-model by Pflüger (2004) has the same production structure as the model by Forslid and Ottaviano (2003). The crucial difference is the form of the upper tier utility function, which is given by $U(X, A)=\alpha \ln X+A$. All income effects are eliminated from manufacturing and channelled into the agricultural sector. The subpreferences for the manufacturing varieties are CES and trade costs are of the iceberg type as in the CP-model. Hence, the demand curves for individual varieties have price elasticity $\sigma$.

In the QLLog-model the indirect utility function is given by $V(\cdot)=Y(\cdot)-\ln P(\cdot)$, so that $V_{Y}=1$, and $V_{P}=-\alpha / P(\cdot)$. The CES price index $P(\lambda, \phi)$ corresponds with the one of the CP model. Applying (5) together with $\lambda=\frac{1}{2}$ and using the expression for $Y(\lambda, \phi)$ and $P(\lambda, \phi)$ from Pflüger (2004) yields the break point $\phi_{b, Q L L o g}$. The location pattern and the welfare properties are now even simpler to determine, since with quasi-linear models preferences: $V_{Y Y}=V_{Y Y Y}=V_{Y P}=V_{Y P P}=V_{Y Y P}=0$. Inserting $\phi=\phi_{b, Q L L o g}$ in eqs. (7) and (14) yields $\Delta_{Q L L o g}<0$ 
and $\Psi_{\text {QLLog }}<0$. This verifies that the QLLog-model exhibits a pitchfork bifurcation (fig. 1b) and features over-agglomeration at the break point.

\subsection{The QLQuad-model}

The QLQuad-model by Ottaviano, Tabuchi and Thisse (2002) differs more profoundly from the two previous frameworks in that it is neither based on CES preferences, nor on iceberg trade costs. However, it is related to the QLLog-model, because it also assumes quasi-linear preferences. The upper-tier utility function is given by $U(X, A)=X+A$ with sub-utility

$$
X=\alpha \int_{i=0}^{N+N^{*}} x_{i} d i-\frac{\beta-\delta}{2} \int_{i=0}^{N+N^{*}} x_{i}^{2} d i-\frac{\delta}{2}\left(\int_{i=0}^{N+N^{*}} x_{i} d i\right)^{2}
$$

Demand curves for the single varieties are linear rather than isoelastic. Imposing the restrictions $\beta>\delta>0$ and $\alpha>0$, these preferences exhibit the "love for variety"-effect. Turning to the supply side, Ottaviano, Tabuchi and Thisse (2002) assume price competition on segmented markets rather than mill pricing, and trade costs are measured in units of the numeráire good by the parameter $\tau$. Producer prices are no longer constant mark-ups over marginal costs.

The QLQuad-model leads to a particularly simple additive separable form of indirect utility $V(\lambda, \phi)=Y(\cdot)-P(\cdot)+\bar{q}_{0}$, where $\bar{q}_{0}>0$ is a constant. Hence, $V_{Y}=1, V_{P}=0$ and all higher order derivatives of $V(\cdot)$ are zero. The impact of prices on indirect utility can be characterised inversely by the consumer surplus, a scalar. This scalar, $P(\lambda, \tau)$, and the income function $Y(\lambda, \tau)$ are quadratic in $\lambda$. Hence, $Y_{\lambda \lambda \lambda}=P_{\lambda \lambda \lambda}=0$. Regarding the shape of the location pattern, due to $V_{P P}=V_{P P P}=0$, eq. (7) reduces to $\Delta_{\text {QLQuad }}=2\left(P_{\lambda \lambda \lambda}+Y_{\lambda \lambda \lambda}\right)=0$. It follows that the bifurcation pattern is a borderline case between tomahawk and pitchfork bifurcation (as illustrated in fig. 1c). Applying (14) yields $\Psi_{\text {QLQuad }}<0$. Hence, the QLQuadmodel implies market over-agglomeration at the break point (Ottaviano and Thisse 2002).

\section{$4 \quad$ Model comparison}

The three models that we have presented as special cases of our framework share the properties $V_{Y Y}=V_{Y Y Y}=V_{Y Y P}=0$ since the indirect utility function is linear in $Y$ in all these cases. However, the marginal utility of income is independent of prices only in the quasi- 
linear models. As a result of income effects in the $X$-sector, $V_{Y}$ is decreasing in $P\left(V_{Y P}<0\right)$ and always below unity $\left(0<V_{Y}<1\right)$ in the CP-model. Table 1 summarises the signs of the utility, price and income responses evaluated at the break point $\tau_{b}\left(\phi_{b}\right)$ for the three models.

[Table 1 about here]

An inspection of this table shows that there is actually just one single case where a derivative has a different sign across models, namely $Y_{\lambda \lambda}$ is positive in the CP- and the QLLog-model, but negative in the QLQuad-model. This wide correspondence of signs is useful for pinning down why these three footloose entrepreneur models differ with respect to their location pattern and their welfare properties.

Focussing first on the location pattern, the shape of the bifurcation of the QLQuad-model is easily rationalised by the fact that the indirect utility $V(Y(\cdot), P(\cdot))$ is linear in both arguments, and that $Y_{\lambda \lambda \lambda}=P_{\lambda \lambda \lambda}=0$ due to the assumption of a quadratic sub-utility for the manufacturing aggregate $X$. To understand why the bifurcation pattern is a tomahawk in the CP-, but a pitchfork in the QLLog-model we use the decomposition of $\Delta(\cdot)$ into parts which entails only direct derivatives $\left(\Lambda_{P}\right.$ and $\Lambda_{Y}=V_{Y} Y_{\lambda \lambda \lambda}$ ) and the term $\Lambda_{Y P}$ that captures the cross-partial derivatives $V_{Y P}$ and $V_{Y P P}$. This term $\Lambda_{Y P}$ is equal to zero in the QLLog-model (as in any quasi-linear model), but it is positive in the CP-model. Furthermore, the two models share the properties $\Lambda_{P}>0, \Lambda_{Y}<0$ and $\left(\Lambda_{P}+\Lambda_{Y}\right)<0$ (see appendix B). Hence, the positive overall sign of $\Delta(\cdot)$ in the CP-case must be due to the fact that the positive term $\Lambda_{Y P}$ compensates the negative term $\left(\Lambda_{P}+\Lambda_{Y}\right)$. Economically speaking, $\Lambda_{Y P}$ represents income effects in the demand for manufactures $X$ which are absent in quasi-linear models. Hence, our analysis suggests that income effects are the cause of the tomahawk shape of the location pattern. ${ }^{9}$

Turning to a comparison of the welfare properties, it seems striking that the CP-model with income effects in the demand for $X$ is the only case where too little agglomeration can occur. However, these income effects can not be the cause for the potential under-agglomeration. To see this, we use $V_{Y Y}=0$ and rewrite eq. (14) as

\footnotetext{
${ }^{9}$ Note, however, that absence of income effects ( $\Lambda_{Y P}=0$ ) does not necessarily imply a pitchfork bifurcation. Although $\left(\Lambda_{P}+\Lambda_{Y}\right)<0$ holds in the QLLog-model, this need not be true in general; e.g. a tomahawk shape follows if $\Lambda_{P}$ is sufficiently strongly positive, whereas $\Lambda_{Y}$ is sufficiently close to zero, which in turn might be due to a low value of $V_{Y}$.
} 


$$
\Psi(\cdot)=(1+\rho)\left(V_{P} P_{\lambda \lambda}+V_{P P} \cdot\left(P_{\lambda}\right)^{2}\right)+V_{Y} Y_{\lambda \lambda}+2 V_{Y P} Y_{\lambda} P_{\lambda} .
$$

where we have set $K \equiv 1$, for convenience. Since $Y_{\lambda}$ and $P_{\lambda}$ have the same sign (see the summary of results in section 2), the third term of (14') which captures income effects, $2 V_{Y P} Y_{\lambda} P_{\lambda}$, must be non-positive as $V_{Y P} \leq 0$. This reveals that income effects in the demand for $X$ exacerbate over-agglomeration rather than leading to under-agglomeration. With respect to the other two terms, it can be shown that $(1+\rho)\left(V_{P} P_{\lambda \lambda}+V_{P P} \cdot\left(P_{\lambda}\right)^{2}\right)<0$ in all models (see appendix B). The term $V_{Y} Y_{\lambda \lambda}$ is negative in the QLQuad-case, so that $\Psi()<$.0 . In the QLLogand the CP-model, we have $V_{Y} Y_{\lambda \lambda}>0$. In the QLLog-model this positive term can never compensate the negative first term, so that $\Psi()<$.0 always holds. In the CP-model, the term $V_{Y} Y_{\lambda \lambda}>0$ may compensate the other two terms in (14'), which are both negative. This requires $\rho$ to be sufficiently small. The potential under-agglomeration in the CP-model must therefore originate in the term $V_{\mathrm{Y}} Y_{\lambda \lambda}$. Taking into account that the marginal utility of income is smaller in the CP-model than in the quasi-linear frameworks, we can identify the underlying cause of the potential under-agglomeration even more precisely. It must be due to the fact that $Y_{\lambda \lambda}$ is sufficiently strongly positive in the CP-model, i.e. that the income function $Y(\cdot)$ is sufficiently strongly convex in $\lambda$ at $\phi_{b}$.

\section{Conclusion}

In this paper we have developed a unifying new economic geography framework that comprehends the class of footloose-entrepreneur models. We have discussed how the results of these models with regard to the shape of the location pattern and the efficiency of equilibrium can be reconciled. Our framework can be used to pin down the properties which explain the differences across models. For example, the core-periphery framework is the only model that features income effects in the demand for manufacturing varieties. We have shown that this distinctive feature can explain why the CP-model exhibits a tomahawk bifurcation. However, it can not explain why the CP-model is the only model which gives rise to underagglomeration.

Although our framework is not applicable to any arbitrary preference structure, it may be used to develop further footloose entrepreneur models and to generalise positive and normative 
results in this model tradition. One insight is that income effects for the differentiated good tend to exacerbate over-agglomeration. Further such results could be derived by making assumptions on the signs of the derivatives of the utility function and the responses of incomes and prices. Moreover, with suitable modifications, this framework may also be applied to other new economic geography models where agglomeration is due to labour mobility.

\section{References}

Baldwin, R., 2001, The core-periphery model with forward-looking expectations, Regional Science and Urban Economics 31, 21-49

Baldwin, R., R. Forslid, P. Martin, G. Ottaviano and F. Robert-Nicoud, 2003, Economic geography and public policy, Princeton University Press

Behrens, K. and Y. Murata, 2006, General equilibrium of monopolistic competition - A new approach, mimeo, CORE, Louváin-la-Neuve

Charlot, S., C. Gaigné, F. Robert-Nicoud and J.-F.Thisse, 2005, Agglomeration and welfare: the core-periphery model in the light of Bentham, Kaldor and Rawls, Journal of Public Economics 90, 325-347.

Dixit, A. and J. Stiglitz, 1977, Monopolistic competition and optimum product diversity, American Economic Review 67, 297-308.

Forslid, R. and Ottaviano, G., 2003, An analytically solvable core-periphery model, Journal of Economic Geography 3, 229 - 240.

Fujita, M., P. Krugman and A.J. Venables, 1999, The spatial economy. Cities, regions, and international trade, MIT Press, Cambridge, Mass.

Fujita, M. und J.-F. Thisse, 2002, Economics of Agglomeration. Cities, Industrial Location and Regional Growth, Cambridge University Press

Guckenheimer, J. and Ph. Holmes, 1983, Nonlinear Oscillations, Dynamical Systems, and Bifurcations of Vector Fields, Springer, New York, Berlin, Heidelberg, Tokyo

Grandmont, J.M., 1988, Nonlinear Difference Equations, Bifurcations and Chaos: An Introduction. Lecture Notes No.5, May, IMSSS-Economics Lecture Notes Series, Institute for Mathematical Studies in the Social Sciences, Stanford, CA

Krugman. P., 1991, Increasing returns and economic geography, Journal of Political Economy 99, 483-499.

Krugman, P., and A. J. Venables, 1995, Globalization and the inequality of nations, Quarterly Journal of Economics 60, 857-880.

Mas-Colell, A., M. D. Whinston and J.R. Green, Microeconomic Theory, Oxford University Press, 1995

Ottaviano, G.I.P. and F. Robert-Nicoud, 2006, The 'genome' of NEG models with vertical linkages: a positive and normative synthesis, Journal of Economic Geography 6, 113139. 
Ottaviano, G.I.P. and J.-F. Thisse, 2002, Integration, agglomeration and the political economics of factor mobility, Journal of Public Economics 83, 429-456.

Ottaviano, G.I.P., T. Tabuchi and J.-F. Thisse, 2002, Agglomeration and trade revisited, International Economic Review 43, 409-435.

Ottaviano, G.I.P. und J.-F. Thisse, 2004 Agglomeration and economic geography, in: J.-F. Thisse und V. Henderson (eds.), Handbook of Regional and Urban Economics: Cities and Geography, volume 4, 2563-2608

Pflüger, M., 2004, A simple, analytically solvable, Chamberlinian agglomeration model, Regional Science and Urban Economics 34, 565-573.

Pflüger, M. and J. Südekum, 2006, Integration, agglomeration and welfare, revised version of IZA Discussion Paper 1326

Robert-Nicoud, F., 2005, The structure of simple 'new economic geography' models (or, on identical twins), Journal of Economic Geography 5, 201-234.

Tabuchi, T., 1998, Agglomeration and dispersion: a synthesis of Alonso and Krugman, Journal of Urban Economics 44, 333-351

Varian, H.R., 1992, Microeconomic Analysis, 3rd ed., W.W. Norton \& Company

Venables, A.J., 1996, Equilibrium location of vertically linked industries, International Economic Review 37, 341-359. 


\section{Appendix A1 CP-model}

(version by Forslid and Ottaviano 2003)

$V(\cdot)=Y(\lambda, \phi) \cdot(P(\lambda, \phi))^{-\mu}$

with $P(\lambda, \phi)=[\lambda+(1-\lambda) \phi]^{\frac{1}{1-\sigma}}$,

$$
Y(\lambda, \phi)=\frac{\rho \mu\left(2 \sigma \phi \lambda+\left(\sigma-\mu+(\mu+\sigma) \phi^{2}\right)(1-\lambda)\right)}{(\sigma-\mu)\left(\sigma \phi \lambda^{2}+\left(\sigma-\mu+(\mu+\sigma) \phi^{2}\right) \lambda(1-\lambda)+\sigma \phi(1-\lambda)^{2}\right)}
$$

$\sigma>1,0<\mu<1,0<\phi<1,0<\lambda<1$. Note: (A3) is a slightly rewritten version of eq. (16) in Forslid and Ottaviano (2003).

Break point determination $\left(\lambda=\frac{1}{2}\right)$ :

$$
-\left(\frac{V_{P}}{V_{Y}}\right)=\frac{\mu \cdot Y(\cdot)}{P(\cdot)}=\frac{Y_{\lambda}}{P_{\lambda}} \quad \Rightarrow \phi_{b, C P}=\frac{(\sigma-\mu)(\sigma-1-\mu)}{(\sigma+\mu)(\sigma-1+\mu)}
$$

$\phi_{b, C P}>0$ due to assumption $(\sigma-1-\mu)>0$ ("no black hole"-cond.); see eq. (26) of Forslid and Ottaviano (2003).

\section{$\underline{\text { Location pattern }}$}

$$
\begin{array}{r}
\Delta_{C P}\left(\frac{1}{2}, \phi_{b, C P}\right)=\frac{64 \mu^{5} \rho(\sigma-1+\mu)(2 \sigma-1)^{3} \cdot \Gamma_{C P}}{(\sigma-\mu)(\sigma-1)^{3}\left(\mu^{2}+\sigma(\sigma-1)\right)^{3}} \cdot(\sigma-1-\mu)>0 \\
\text { where } \Gamma_{C P} \equiv\left(\frac{\mu^{2}+\sigma(\sigma-1)}{(\mu+\sigma)(\mu+\sigma-1)}\right)^{\frac{\mu}{(\sigma-1)}}>0, \rho \equiv L / K>0
\end{array}
$$

Welfare

$$
\begin{aligned}
\Psi_{C P}\left(\frac{1}{2}, \phi_{b}^{C P}\right)= & \frac{8 \mu^{3} \rho(\sigma-1-\mu)(2 \sigma-1) \cdot \Gamma_{C P}}{(\sigma-\mu)(\sigma-1)^{2}\left(\mu^{2}+\sigma(\sigma-1)\right)^{2}} \cdot[2 \sigma(\sigma-1)-\mu(2 \rho(2 \sigma-1)-1)] \\
& \Rightarrow \Psi_{\mathrm{QLLog}}<0 \Leftrightarrow \rho>\frac{\mu+2 \sigma(\sigma-1)}{2 \mu(\sigma-1)}
\end{aligned}
$$




\section{Appendix A2 QLLog-model (Pflüger 2004)}

$V(\cdot)=Y(\cdot)-\alpha \cdot \ln P(\cdot)$

with $Y(\lambda, \phi)=\frac{\alpha}{\sigma}\left[\frac{\rho+\lambda}{\lambda+(1-\lambda) \phi}+\frac{\phi(\rho+1-\lambda)}{\phi \lambda+(1-\lambda)}\right], \quad P(\lambda, \phi)=\kappa[\lambda+(1-\lambda) \phi]^{\frac{1}{1-\sigma}}$

Break point determination $\left(\lambda=\frac{1}{2}\right)$ :

$$
-\left(\frac{V_{P}}{V_{Y}}\right)=\frac{\alpha}{P(\cdot)}=\frac{Y_{\lambda}}{P_{\lambda}} \Rightarrow \phi_{b, Q L L o g}=\frac{\sigma(2 \rho-1)-2 \rho}{\sigma(3+2 \rho)-2(1+\rho)}
$$

\section{$\underline{\text { Location pattern }}$}

$$
\Delta_{\text {QLLog }}(\cdot)=-\frac{64 \alpha(2 \sigma-1)^{3}}{(1+2 \rho)^{3}(\sigma-1)^{4}}<0 \quad \text { (A10) (cf. Pflüger 2004: } 569 \text { and footnote 10). }
$$

$\underline{\text { Welfare }}$

$$
\Psi_{\text {QLLog }}(\cdot)=-\frac{2 \alpha(2 \sigma-1)}{(1+2 \rho)(\sigma-1)^{3}}<0
$$

Note: By setting eq. (13) equal to zero, one obtains $\phi_{p \text {,QLLog }}=(2 \rho-1) /(2 \rho+3)>\phi_{b \text {,QLLog }}$, the 'social break point' of the QLLog-model (see Pflüger and Südekum 2006). 


\section{Appendix A3 QLQuad-model (Ottaviano, Tabuchi and Thisse 2002)}

$$
\begin{aligned}
& Y(\cdot)=\frac{b F+c K}{4 F^{2}(2 b F+c K)^{2}}\left[(2 a F+\tau c K(1-\lambda))^{2}\left(\frac{L}{2}+\lambda K\right)+(2 a F-2 \tau b F-\tau c K(1-\lambda))^{2}\left(\frac{L}{2}+(1-\lambda) K\right)\right] \\
& P(\cdot)=\frac{K}{8 F^{2}(c K+2 b F)^{2}}\left[\begin{array}{c}
c^{3} K^{3} \tau^{2}(1-\lambda) \lambda+c^{2} K^{2} \tau F(1-\lambda)(b \tau(4+\lambda)-8 a)- \\
8 c K F^{2}\left(a^{2}+2 a b \tau(1-\lambda)-b^{2} \tau^{2}(1-\lambda)\right)-4 b F^{3}\left(3 a^{2}+2 a b \tau(1-\lambda)-b^{2} \tau^{2}(1-\lambda)\right)
\end{array}\right]
\end{aligned}
$$

where $a, b, c, F$ are primitives of the model that are originally labelled $\phi, \mathrm{L}$ and $\mathrm{A}$. We have changed these symbols to avoid confusion (we have substituted out prices (eqs. (7) and (10) into the expression for consumer surplus (page 439) in the analysis of Ottaviano and Thisse 2002)).

Break point determination $\left(\lambda=\frac{1}{2}\right)$ : $\quad$ (cf. Ottaviano and Thisse 2002: 420)

$$
-\left(\frac{V_{P}}{V_{Y}}\right)=1=\frac{Y_{\lambda}}{P_{\lambda}} \quad \Rightarrow \tau_{b, Q L Q}=\frac{4 a F(3 b F+2 c K)}{2 b F(3 b F+3 c K+c L)+c^{2} K(L+K)}
$$

$\underline{\text { Welfare }}$

$$
\begin{aligned}
& \Psi(\cdot)=-\frac{4 a^{2} c^{2} K^{2}(L+K)(c K+b F)(2 c K+3 b F)(2 c K+5 b F)}{(c K+2 b F)^{2}\left(c^{2} K(L+K)+2 b c F(L+3 K)+6 b^{2} F^{2}\right)^{2}}<0 \\
& \tau_{p, Q L Q}=\frac{16 a F(3 b F+c K)}{8 b F(3 b F+2 c K+c L)+3 c^{2} K(L+K)}<\tau_{b, Q L Q}
\end{aligned}
$$




\section{Appendix B Model comparison}

\section{$\underline{\text { CP-model }}$}

$$
\begin{aligned}
& \Lambda_{P}=\frac{16 \mu^{5} \rho(2 \sigma-1)^{3}(2(\sigma-1)-\mu)(\sigma-1-\mu) \cdot \Gamma_{C P}}{(\sigma-\mu)(\sigma-1)^{3}\left(\mu^{2}+\sigma(\sigma-1)\right)^{3}}>0, \quad \Gamma_{C P} \equiv\left(\frac{\mu^{2}+\sigma(\sigma-1)}{(\mu+\sigma)(\mu+\sigma-1)}\right)^{\frac{\mu}{(\sigma-1)}} \\
& \Lambda_{Y}=-\frac{96 \mu^{5}(2 \sigma-1)^{2} \cdot \Gamma_{C P}}{(\sigma-\mu)(\sigma-1)^{2}\left(\mu^{2}+\sigma(\sigma-1)\right)^{2}}<0 \\
& \left(\Lambda_{P}+\Lambda_{Y}\right)=-\frac{16 \mu^{5} \rho(2 \sigma-1)^{2}(2 \sigma(\sigma+2 \mu)-5 \mu-2)(\sigma-1+\mu) \cdot \Gamma_{C P}}{(\sigma-\mu)(\sigma-1)^{3}\left(\mu^{2}+\sigma(\sigma-1)\right)^{3}}<0 \\
& \Lambda_{Y P}=\frac{48 \mu^{5} \rho(2 \sigma-1)^{2}(2 \sigma(\sigma-1)-\mu)(\sigma-1+\mu) \cdot \Gamma_{C P}}{(\sigma-\mu)(\sigma-1)^{3}\left(\mu^{2}+\sigma(\sigma-1)\right)^{3}}>0 \\
& \Rightarrow 2\left[\left(\Lambda_{P}+\Lambda_{Y}\right)+\Lambda_{Y P}\right]=\Delta_{C P}(\cdot), \text { see eq. }(\mathrm{A} 5) \\
& (1+\rho)\left(V_{P} P_{\lambda \lambda}+V_{P P} \cdot\left(P_{\lambda}\right)^{2}\right)=-\frac{8 \mu^{4} \rho(1+\rho)(2 \sigma-1)^{2}(\sigma-1-\mu) \cdot \Gamma_{C P}}{(\sigma-\mu)(\sigma-1)^{2}\left(\mu^{2}+\sigma(\sigma-1)\right)^{2}}<0 \\
& \Rightarrow(1+\rho)\left(V_{P} P_{\lambda \lambda}+V_{P P} \cdot\left(P_{\lambda}\right)^{2}\right)+V_{Y} Y_{\lambda \lambda}+2 V_{Y P} Y_{\lambda} P_{\lambda}=\Psi_{C P}(\cdot), \text { see eq. }(\mathrm{A} 6) \\
& V_{Y} Y_{\lambda \lambda}=\frac{8 \mu^{3} \rho(2 \sigma-1)^{2} \cdot \Gamma_{C P}}{(\sigma-\mu)(\sigma-1)\left(\mu^{2}+\sigma(\sigma-1)\right)}<0 \\
& V_{Y P} P_{\lambda} Y_{\lambda}=-\frac{(\sigma-\mu)(\sigma-1)^{2}\left(\mu^{2}+\sigma(\sigma-1)\right)^{2}}{(\sigma-1) \cdot \Gamma_{C P}}
\end{aligned}
$$

\section{QLLog-model}

$$
\begin{aligned}
& \Lambda_{P}=\frac{16 \alpha(2 \sigma-1)^{3}}{(2 \rho+1)^{3}(\sigma-1)^{4}}>0 \quad \Lambda_{Y}=-\frac{48 \alpha(2 \sigma-1)^{3}}{(2 \rho+1)^{3}(\sigma-1)^{4}}<0 \quad \Lambda_{Y P}=0 \\
& \Rightarrow 2\left(\Lambda_{P}+\Lambda_{P}\right)=\Delta_{Q L L o g}(\cdot)<0 \text {, see eq. (A10) } \\
& (1+\rho)\left(V_{P} P_{\lambda \lambda}+V_{P P} \cdot\left(P_{\lambda}\right)^{2}\right)=-\frac{4 \alpha(2 \sigma-1)^{2}}{(2 \rho+1)(\sigma-1)^{3}}<0 \quad V_{Y} Y_{\lambda \lambda}=\frac{8 \alpha(2 \sigma-1)}{(2 \rho+1)(\sigma-1)^{2}}>0 \\
& \Rightarrow(1+\rho)\left(V_{P} P_{\lambda \lambda}+V_{P P} \cdot\left(P_{\lambda}\right)^{2}\right)+V_{Y} Y_{\lambda \lambda}=\Psi_{Q L L o g}(\cdot) \text {, see eq. (A11) }
\end{aligned}
$$


Table 1: Properties of the specific models (at $\lambda=\frac{1}{2}$ and $\tau=\tau_{b}$ )

\begin{tabular}{|c|c|c|c|}
\hline & CP & QLLog & QLQuad \\
\hline $\mathbf{V}_{\mathbf{Y}}$ & $0<\mathrm{V}_{\mathrm{Y}}<1$ & 1 & 1 \\
\hline $\mathbf{V}_{\mathbf{Y P}}=\mathbf{V}_{\mathbf{P Y}}$ & 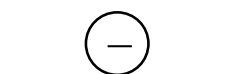 & $\mathbf{0}$ & $\mathbf{0}$ \\
\hline $\mathbf{V}_{\mathbf{Y P P}}=\mathbf{V}_{\mathbf{P Y Y}}$ & + & $\mathbf{0}$ & $\mathbf{0}$ \\
\hline $\mathbf{V}_{\mathbf{P}}$ & $\Theta$ & $\theta$ & -1 \\
\hline $\mathbf{V}_{\mathbf{P P}}$ & + & + & $\mathbf{0}$ \\
\hline $\mathbf{V}_{\text {PPP }}$ & $\Theta$ & $\Theta$ & $\mathbf{0}$ \\
\hline $\mathbf{P}_{\lambda}$ & $\theta$ & $\theta$ & $\theta$ \\
\hline $\mathbf{P}_{\lambda \lambda}$ & $\oplus$ & + & + \\
\hline $\mathbf{P}_{\lambda \lambda \lambda}$ & 0 & 0 & 0 \\
\hline $\mathbf{Y}_{\lambda}$ & - & 0 & 0 \\
\hline $\mathbf{Y}_{\lambda \lambda}$ & + & + & 0 \\
\hline $\mathbf{Y}_{\lambda \lambda \lambda}$ & 0 & 0 & $\mathbf{0}$ \\
\hline
\end{tabular}




\section{Figure 1 Characteristics of different agglomeration models}

a) Cobb-Douglas/CES model (Krugman 1991, Forslid and Ottaviano 2003) tomahawk bifurcation, over- or under-agglomeration at $\phi_{b}$

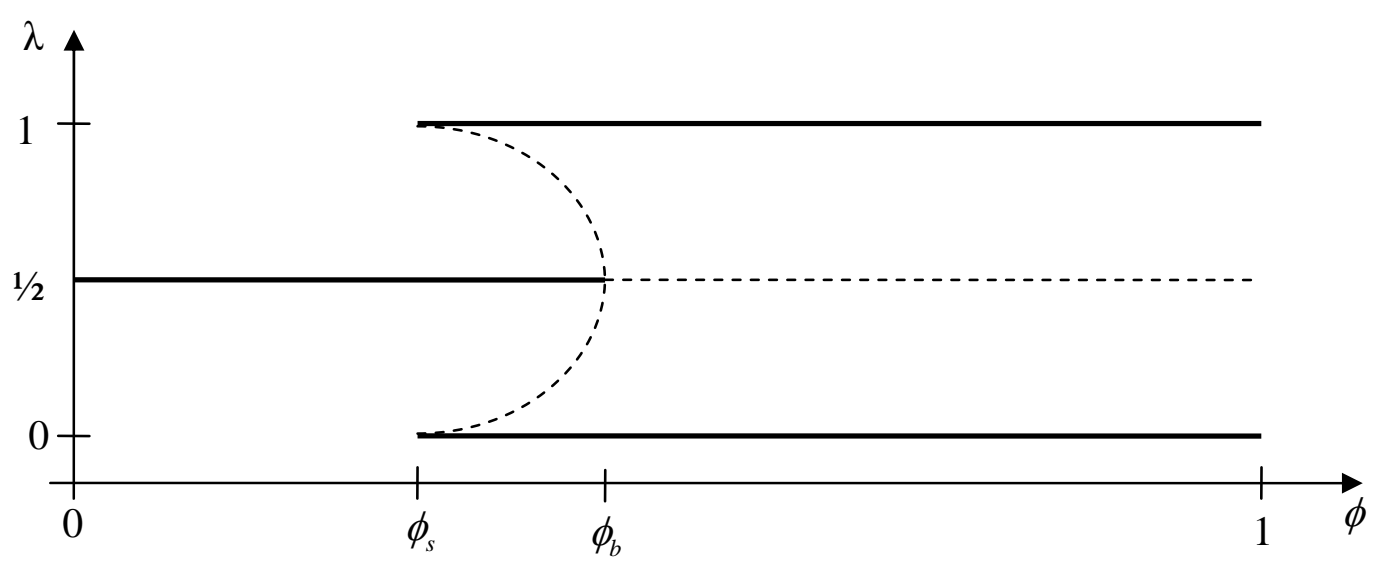

b) Quasi-linear logarithmic model (Pflüger 2004) pitchfork bifurcation, over-agglomeration at $\phi_{b}$

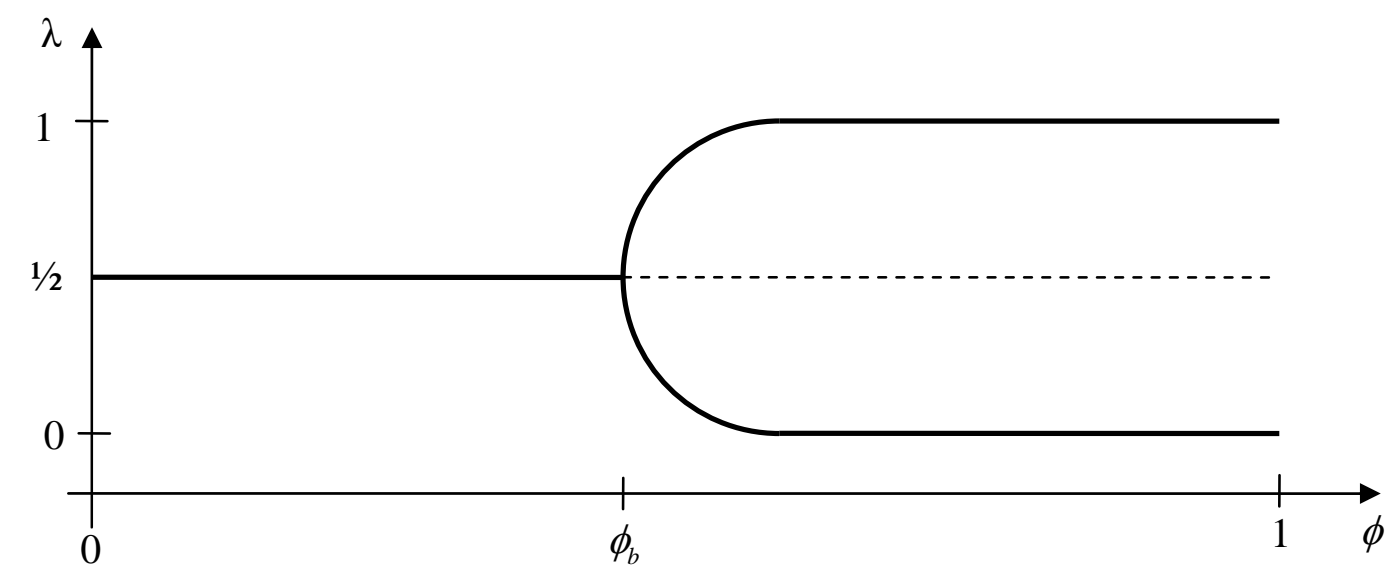

c) Quasi-linear quadratic model (Ottaviano, Tabuchi and Thisse 2002) borderline bifurcation type, over-agglomeration at $\phi_{b}$

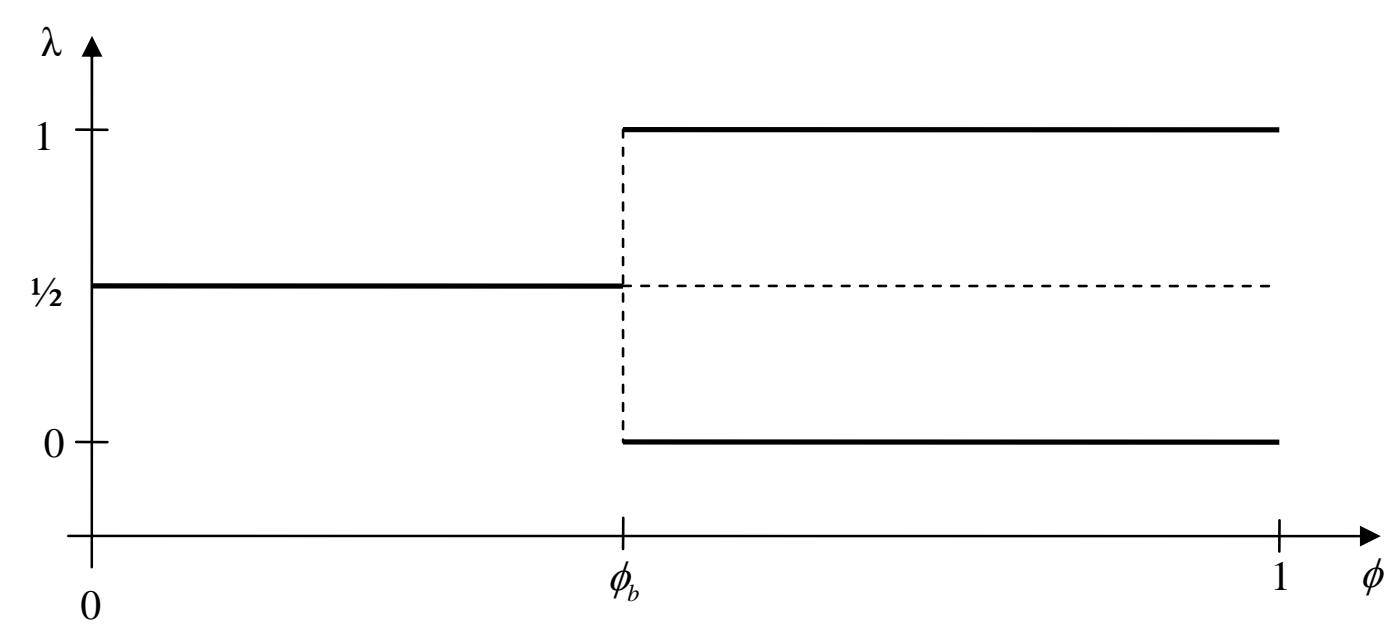


Figure 2 The shape of the social welfare function for different trade costs

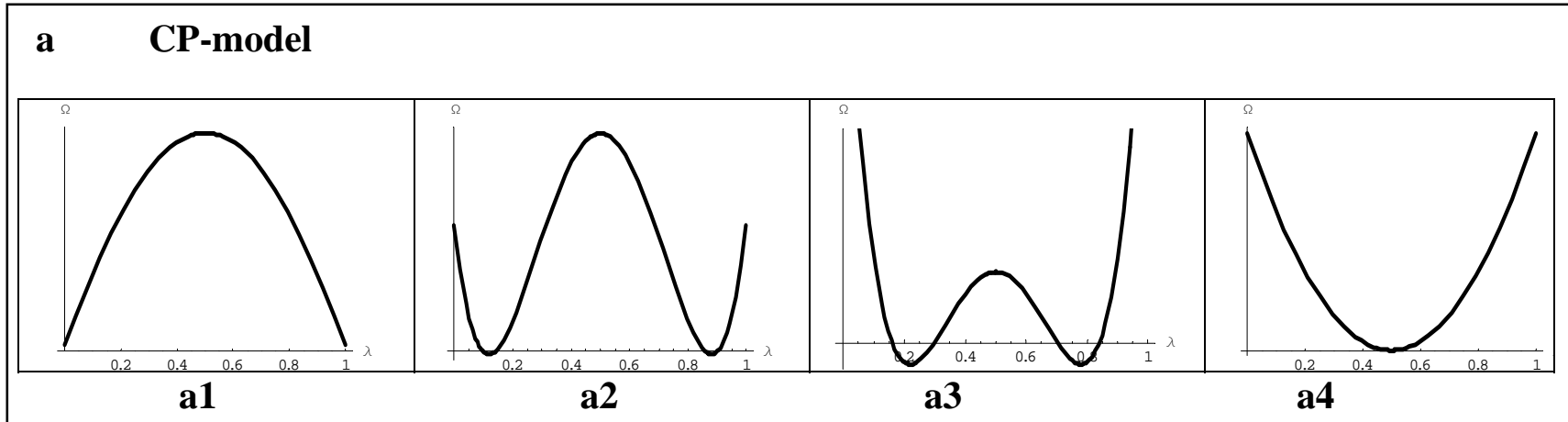

b QLLog-model

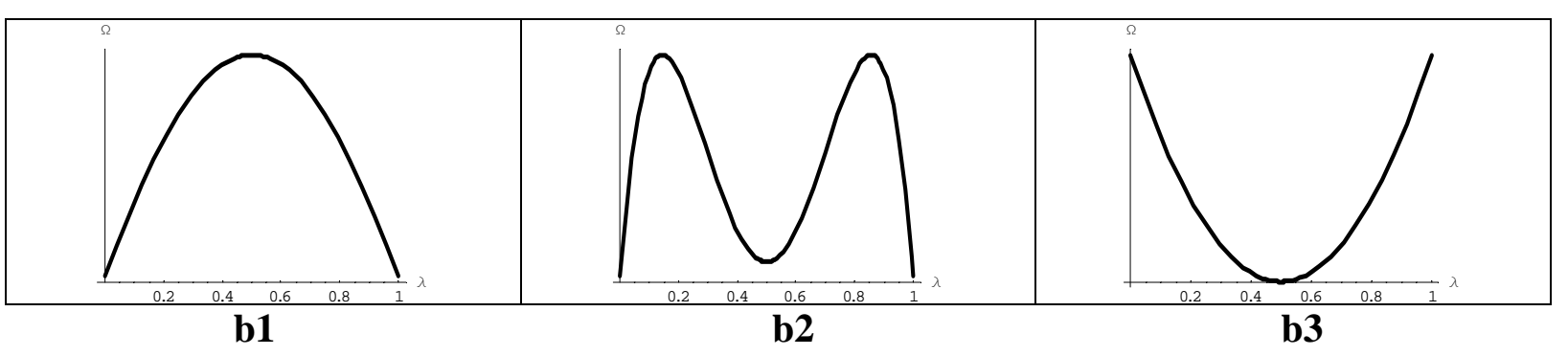

c QLQ-model

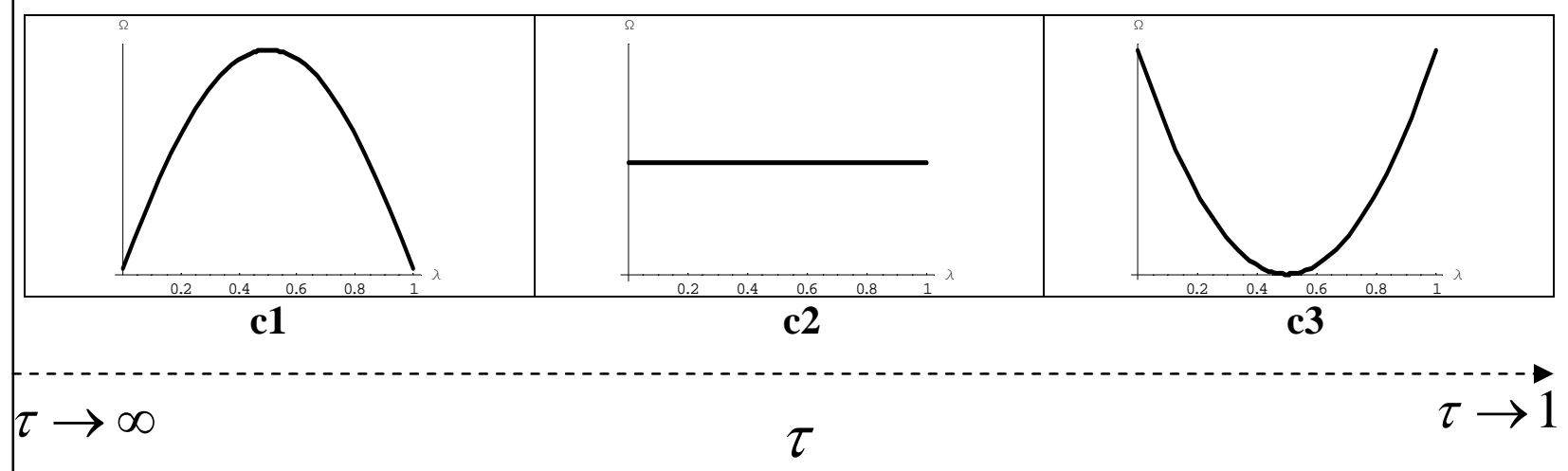

